The University of Southern Mississippi

The Aquila Digital Community

Faculty Publications

7-26-1993

\title{
Driven Diffusion, Kawasaki Dynamics, Mixing, and Spatial Ordering in an Interacting Lattice-Gas
}

Yan He

University of Southern Mississippi

Ras B. Pandey

University of Southern Mississippi, ras.pandey@usm.edu

Follow this and additional works at: https://aquila.usm.edu/fac_pubs

Part of the Physics Commons

\section{Recommended Citation}

He, Y., Pandey, R. B. (1993). Driven Diffusion, Kawasaki Dynamics, Mixing, and Spatial Ordering in an Interacting Lattice-Gas. Physical Review Letters, 71(4), 565-568.

Available at: https://aquila.usm.edu/fac_pubs/6506

This Article is brought to you for free and open access by The Aquila Digital Community. It has been accepted for inclusion in Faculty Publications by an authorized administrator of The Aquila Digital Community. For more information, please contact Joshua.Cromwell@usm.edu. 


\title{
Driven Diffusion, Kawasaki Dynamics, Mixing, and Spatial Ordering in an Interacting Lattice Gas
}

\author{
Yan He and Ras B. Pandey \\ The Program in Scientific Computing, Department of Physics and Astronomy. \\ University of Southern Mississippi, Hattiesburg, Mississippi 39406-5046
}

(Received 18 January 1993)

\begin{abstract}
Kawasaki dynamics is used to study the transport properties of a nonequilibrium steady state system of interacting lattice gas of oppositely charged particles in a linear gradient field in two dimensions. The rms displacements show unusual nondiffusive transport. The effective conductivity varies with the temperature which deviates from the Arrhenius law and depends on the range of interaction. Density of a fully mixed state decays with temperature with a power law. Onset of spatial ordering occurs in a certain temperature range at a fixed range of interaction.
\end{abstract}

PACS numbers: $64.60 . \mathrm{Ht}, 05.40 .+\mathrm{j}, 05.70 . \mathrm{Ln}, 68.35 . \mathrm{Fx}$

Transport of particles (charged or neutral atoms and molecules) at the surfaces plays a very important role in understanding the kinetics of adsorption and desorption and the evolution of spatial ordering in chemisorbed or physisorbed surfaces in the vapor deposition and coating processes. Several experiments have been carried out in recent years to elucidate how diffusion of the adsorbates affects the ordering, i.e., CO diffusion, on $\mathrm{Pt}(111)$ [1], hydrogen and deuterium on tungsten [2], and nickle [3] surfaces. Numerous theoretical investigations have been carried out on understanding the ordering via simple model systems such as lattice gas and Ising models with nearest neighbor and next nearest neighbor interactions [4,5]. Relatively few attempts have been made to study both the transport and the ordering simultaneously in particular to understand how the spatial ordering settles as the particles diffuse on the surface [6,7]. Even with the nearest neighbor $(\mathrm{nn})$ interaction the simple lattice gas models [6-8] show a variety of unusual transport properties such as subdiffusive transport, metastability associated with the onset of domain growth, and reentrant global transport behavior as a function of coverage and temperature. The phase transitions associated with the spatial ordering in most of these model systems are well studied [4,5]. It would be interesting to investigate the effect of an external biased field on both transport properties and spatial ordering for the following reasons: (1) It is easier to investigate the global transport properties such as conductivity in the presence of field. (2) Since a system is driven out of equilibrium due to biased field, it would be interesting to see if a stable special spatial ordering sets in the nonequilibrium steady state. In this Letter we attempt to address these issues for an interacting lattice gas system.

Several attempts have been recently made to understand the transport and spatial ordering in nonequilibrium steady state systems [9-13]. Most of these studies are limited to nearest neighbor lattice gas and many of these studies $[9,10]$ focus primarily on phase transitions in the presence of a global biased field constant at all lattice sites. In this Letter, we consider an interacting lattice gas model of a binary mixture of oppositely charged particles with conserved number and charge where a Coulomb-like interaction is extended up to fourth neighbors. Furthermore, instead of considering the same field at all sites $[9,10]$, we use a linear gradient field which usually develops due to the distribution of mobile ions in fluid mixtures [13]. We find several new results: unusual nondiffusive transport behavior, non-Arrhenius dependence of conductivity on temperature, onset of spatial ordering, domain formation, and a mixing-demixing phase transition [5,6].

We consider a square lattice. Half of the lattice sites are randomly occupied by particles. Each particle is assigned a unit charge density while each empty site a negative unit charge density such that the total charge of the whole lattice is zero. A linear gradient field is set up across the lattice. The interaction energy is given by

$$
H=\sum_{i j} \rho_{i} \rho_{j} / r_{i j}+\sum_{i_{x}} B_{0}\left(1-i_{x} / L_{x}\right) \rho_{i},
$$

where $\rho_{i}$ is the charge density of an empty site or particle at site $i, r_{i j}$ is the distance between $i$ and $j$ sites, $i_{x}$ is the $x$ coordinate of site $i, L_{x}$ is the linear length along the $x$ axis, and $B_{0}$ is the field strength which is chosen as unity. We are restricted here to a highly truncated range of interaction, i.e., up to fourth neighbor $(4 n)$; however, this is larger than the range considered in previous studies [8-10]. We use the Metropolis algorithm [14] to move a particle to its neighboring empty site and a periodic boundary condition is imposed across the boundaries. For the nearest neighbor interaction, in the absence of field $\left(B_{0}=0\right)$, our model is reduced to a nn antiferromagnetic Ising model [15] where an "up" spin corresponds to a particle while a "down" spin to an empty site. The mechanism of particles' hopping corresponding to a nearest neighbor spin exchange thus represents the Kawasaki dynamics [16].

Figure 1 shows a typical variation of energy with time at temperatures $T=0.70$ and 1.00 (in units of the Boltzmann constant $k_{B}$ ) for a various range of interactions. The energy decays continuously with time at all ranges of interaction and temperatures, except at $T=0.70$ with the nearest neighbor interaction for which the energy saturates. We shall concentrate here primari- 


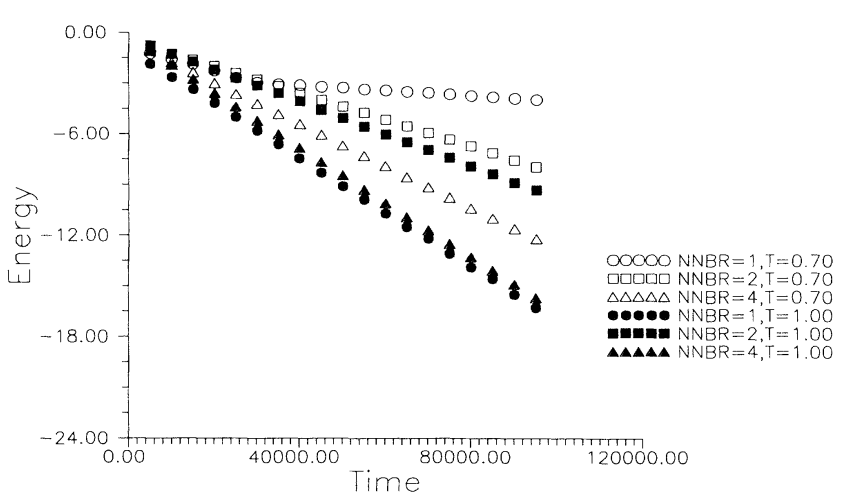

FIG. 1. Energy versus time for the nearest neighbor (O), next nearest neighbor $(\square)$, and fourth neighbor $(\triangle)$ interactions. Filled symbols are at the temperature $T=0.70$ while the unfilled symbols are at $T=1.00$; sample size $60 \times 60$ with time steps up to $10^{5}$ Monte Carlo steps (MCS) were used.

ly on nonequilibrium configurations for which the energy does not approach its equilibrium value as the system is constantly driven out of equilibrium.

Plots for the variation of the $x$ and $y$ components of the rms displacement of each particle (tracer) with time on a $\log -\log$ scale are presented in Fig. 2. A linear fit of the data points especially in the long time regime suggests a power-law behavior of the tracer's diffusion, i.e., $R_{\mathrm{tr}} \sim t^{k}$. Since the gradient field is in the $x$ direction, one would expect a driftlike asymptotic behavior of the $x$ component of the rms displacement of each particle. However, we do not observe a driftlike behavior. The effective exponent $k$ for the variation of the $x$ component of the rms displacement with time seems to depend on the range of interaction and possibly on the temperature. For example, at $T=0.70, k=0.68 \pm 0.2$ [next nearest neighbor (nnn)] and $0.55 \pm 0.02(4 \mathrm{n})$, and at $T=1.00, k=0.53 \pm 0.02$ $(\mathrm{nn}), 0.72 \pm 0.02(\mathrm{nnn})$, and $0.72 \pm 0.02(4 \mathrm{n})$. The $y$ component of $R_{\mathrm{tr}}$, on the other hand, shows a diffusionlike behavior with the effective exponent $k$ around $\frac{1}{2}$ (within the range of 0.47 to 0.52 ). The simulation is performed with different sample sizes in order to see the effects of the finite lattice. Figure 3 shows a typical variation of the rms displacements of each particle with two different samples and, as we see, the results remain qualitatively the same. We have also studied in detail the temporal variation of the center of mass of the particles which is defined as $R_{\text {c.m. }}=\sum_{i} r_{i} / N_{p}$, where $r_{i}$ is the position of the $i$ th particle, and the summation extends over all $N_{p}$ particles. The $x$ and $y$ components of the rms displacement $R_{\text {c.m. }}$ of the center of mass of the particles shows an expected collective transport behavior: The $x$ component of $R_{\text {c.m. }}$ shows a drift, while the $y$ component fluctuates around its center of mass which shows a very slow drift. A power-law dependence of the center of mass on time for the collective motion of particles is also discussed by van Beijeren [17] but in a different context.

We have visually studied the spatial distribution of par- (a)

(b)
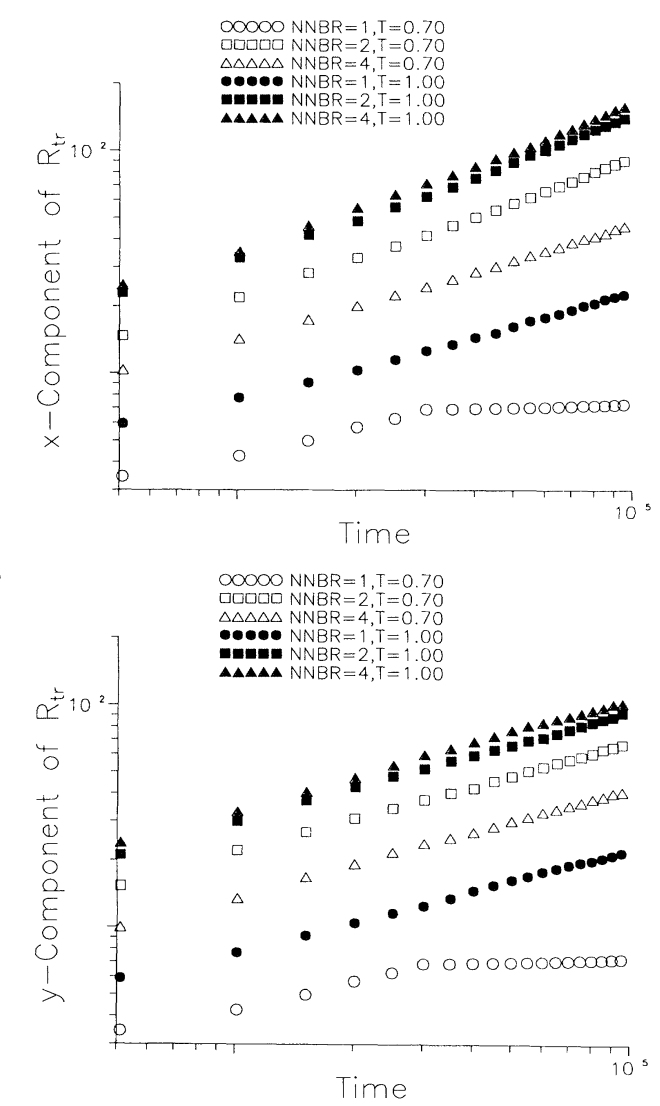

FIG. 2. Variation of the $x$ and $y$ components, in (a) and (b), respectively, of the rms displacement of each particle on a loglog scale. Same statistics as in Fig. 1.

ticles in order to investigate ordering, domain formation, and spinodal decomposition [5]. We have analyzed in detail the growth and decay of clusters [18] formed by particles, holes, and perfectly mixed configurations in which a particle is completely surrounded by neighboring holes and vice versa. With the nearest neighbor and fourth neighbor interactions, we observe the growth of domains of a nearest neighbor antiferromagnetic (AFM) ordering in which a particle is completely surrounded by neighboring holes and vice versa. Note that the size of domains represents the degree of mixing here. In steady state at high temperatures, we see isolated clusters which grow in size as we reduce the temperature. Thus, as a function of temperature we observe a percolationlike transition [18] from isolated AFM clusters to an infinite AFM cluster. The onset of incipient spanning AFM clusters occurs at a critical temperature $\left[T_{\mathrm{cp}} \simeq 1.15(\mathrm{nn}), T_{\mathrm{cp}} \simeq 0.72(4 \mathrm{n})\right.$.] Spectacular domain growth appears with the next nearest neighbor interaction as we vary the temperature (see Fig. 4). We observe a transition from a disorder phase at $T=1.00$ to an ordered (strip) phase at $T=0.30$ and then to a domain of strips at low temperatures ( $T$ $=0.50-0.05$ ).

The concentration of particles in the perfectly mixed 
(a)

(b)
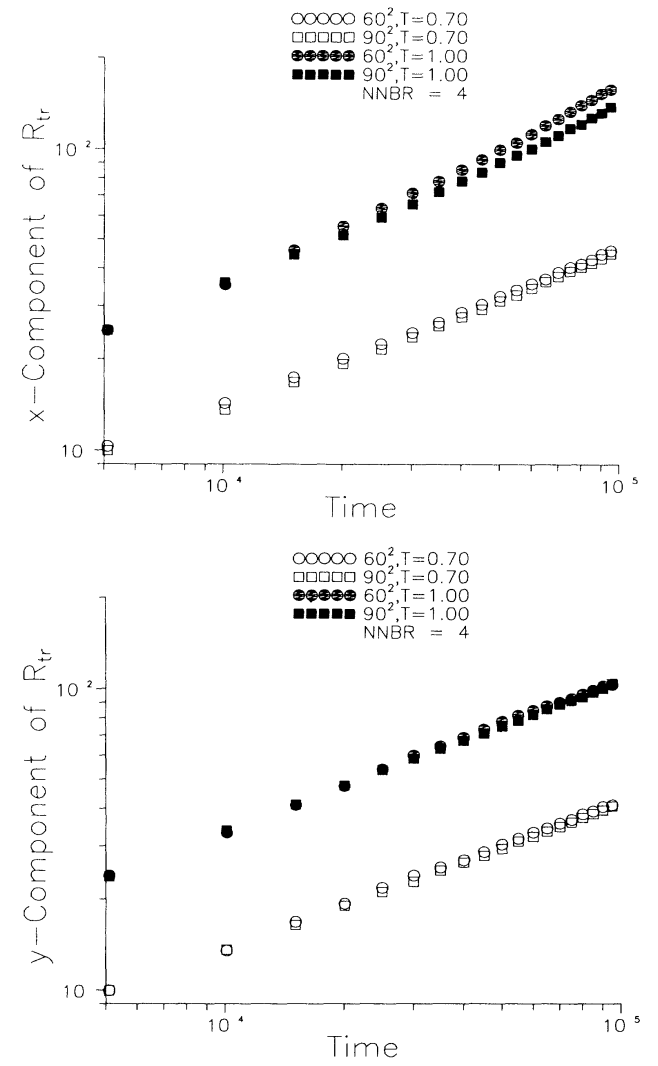

FIG. 3. A typical variation of the rms displacements of each particle with two different samples $[x$ component (a) and $y$ component (b)].

microstate varies with the temperature. A typical plot of the concentration of fully mixed particles $p_{f}$ versus temperature is presented in Fig. 5. From the continuous decay of $p_{f}$, we identify a critical temperature $\left(T_{c} \sim 0.80\right)$ where $p_{f} \rightarrow 0$. A $p_{f}$ versus $T-T_{c}$ plot on a log-log scale gives an excellent linear fit,

$$
p_{f} \sim\left(T-T_{c}\right)^{\beta},
$$

with the exponent $\beta$ about $\frac{1}{2}$. It is tempting to characterize $p_{f}$ as a staggered magnetization although it does not saturate at $T<T_{c}$. Furthermore, this system is not in thermal equilibrium in contrast to second order phase transitions in ordinary AFM [15] where $\beta$ is a well defined exponent for the order parameter. However, the system does not mix above $T_{c}$ on the global scale, and the perfect mixing does not appear at very low temperatures but near $T_{c}$, where $p_{f}$ becomes maximum (see Fig. 5).

For our driven system, it is rather straightforward to estimate the effective conductivity [13] in its steady state. From the flux $Q$ of the particles along the field direction, one can evaluate the current density $j$,

$$
j=\left(1 / L_{y}\right) d Q / d t \text {. }
$$

In the steady state the flux $Q$ of particles going into the (a)
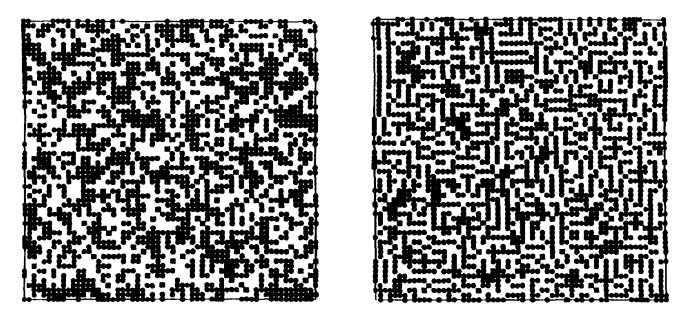

(b)

(c)
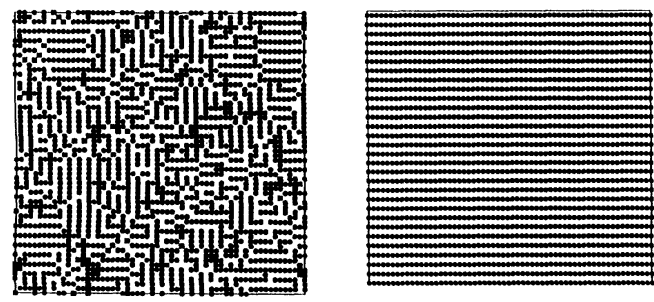

(d)

(e)
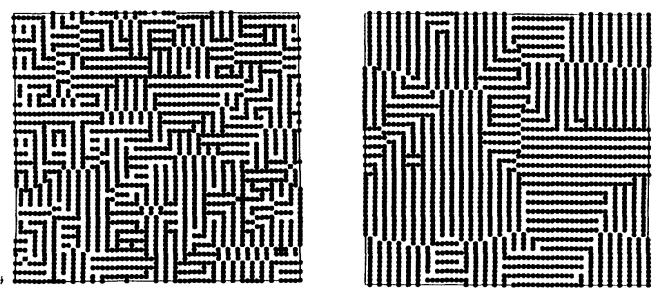

(f)

FIG. 4. Snapshot of the configurations for the next nearest neighbor interaction in steady state (at $10^{5} \mathrm{MCS}$ ) at temperatures $T=10.00$ (a), 1.00 (b), 0.50 (c), 0.30 (d), 0.10 (e), and 0.05 (f); sample size $60 \times 60$ was used. A particle is shown by (•) and a hole by ( ) except in a perfectly mixed microstate in which either a particle surrounded by all neighboring holes or a hole surrounded by all neighboring particles is shown by (ש).

system at one end (i.e., toward the high field) is equal to the flux of particles leaving the system at the opposite end (zero field) along the $x$ direction. Excellent linear plots of our $Q$ versus $t$ data show that the steady state is achieved in rather short time in this model. Using $j=\sigma E_{g}$, we can estimate the effective conductivity $\sigma$ at a fixed temperature; $E_{g}$ is the gradient field which varies as $1 / x$ along the $x$ direction [see Eq. (1)]. Ordinarily, the conductivity $\sigma$ shows an Arrhenius dependence on the temperature, i.e.,

$$
\sigma=A \exp \left(-E_{a} / T\right)
$$

where $A$ is a constant and $E_{a}$ is the activation energy. Therefore, a plot of $\log (\sigma)$ versus $1 / T$ should be linear. Figure 6 shows such a plot for the $n n$, nnn, and $4 n$ interactions. In a very limited temperature regime, a linear decay seems to emerge for the $n n$ and $4 n$ interactions. Such a linear regime is hard to identify with the nnn interaction, which suggests a deviation from the Arrhenius dependence.

In summary, we have presented a simple nonequilibrium steady state model of an interacting lattice gas of a 
(a)

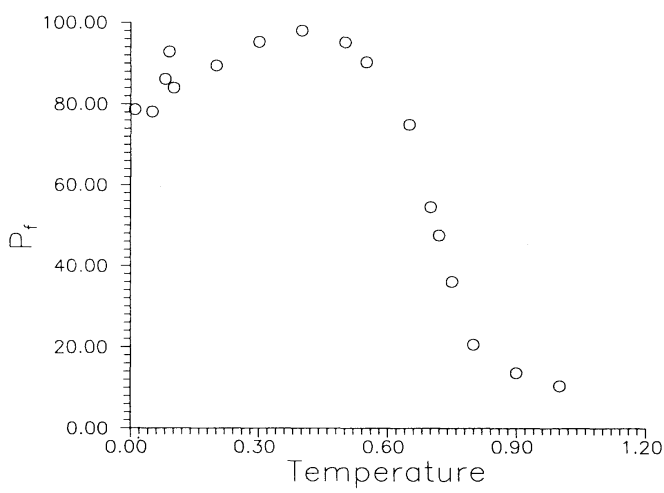

(b)

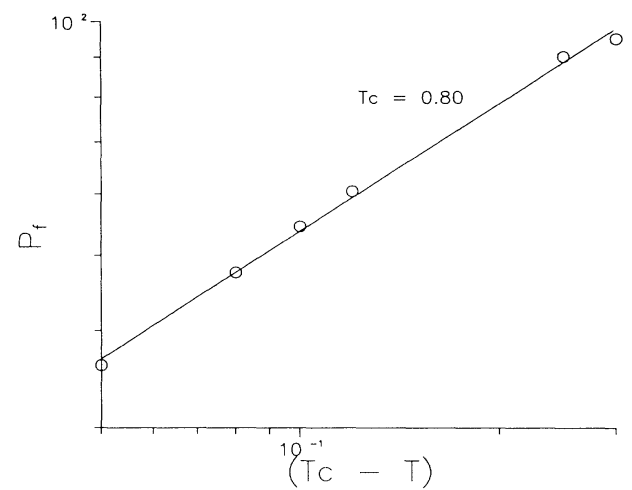

FIG. 5. (a) Fraction of particles $p_{f}$ in a fully mixed state versus temperature $(T)$ for the fourth neighbor interaction in steady state. (b) $p_{f}$ versus $T_{c}-T$ on a $\log -\log$ scale in the critical regime near $T_{c}$. Same statistics as in Fig. 1.

binary mixture in a linear gradient field with Kawasaki dynamics. The range of interaction plays an important role in the spatial ordering: As a function of temperature, a mixing-demixing transition occurs with the nn and $4 \mathrm{n}$ interactions, while special spatial ordering and growth and decay of striplike domains appear with the nnn. Along the field direction, we find that the collective motion of the particles (i.e., their center of mass) is faster (driftlike) than each particle (superdiffusive). The conductivity depends on the temperature and the range of interaction, and evidence for its deviation from the Arrhenius dependence is found. Detailed study on the ordering and transport will be published elsewhere.

The authors would like to thank the Mississippi Center for Supercomputing Research for the support of computational resources. Partial support from a NSF EPSCoR grant is also acknowledged.

[1] J. E. Reutt-Robey, D. J. Doren, Y. J. Chabal, and S. B.

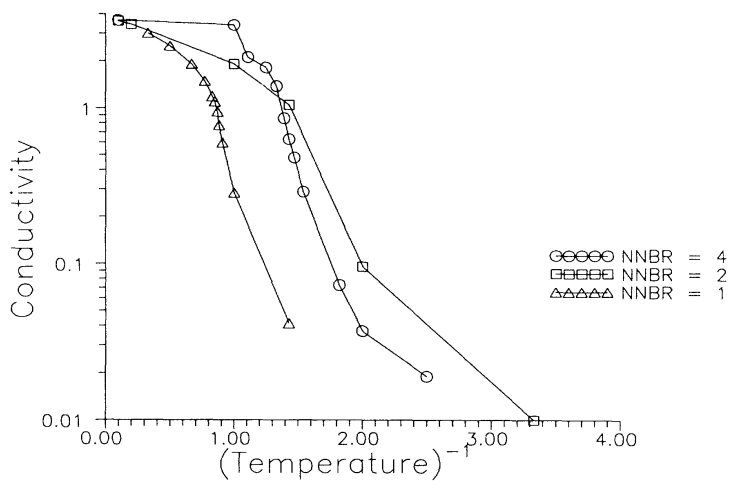

FIG. 6. Conductivity versus inverse temperature on a semilogarithmic scale for the $n n, n n n$, and $4 n$ interactions with the same statistics as in Fig. 1.

Christman, Phys. Rev. Lett. 61, 2778 (1988).

[2] D. S. Choi, C. Uebing, and R. Gomer, Surf. Sci. 259, 139 (1991); C. Uebing and R. Gomer, ibid. 259, 151 (1991).

[3] T.-S. Lin and R. Gomer, Surf. Sci. 255, 41 (1991).

[4] K. K. Chin and D. P. Landau, Phys. Rev. B 36, 275 (1987); T. Aukrust, M. A. Novotny, P. A. Rikvold, and D. P. Landau, Phys. Rev. B 41, 8772 (1990).

[5] J. D. Gunton, M. San Miguel, and P. S. Sahni, in Phase Transitions and Critical Phenomena, edited by C. Domb and J. L. Lebowitz (Academic, New York, 1983), Vol. 8.

[6] A. Sadiq and K. Binder, Surf. Sci. 128, 350 (1983); J. W. Evans and D. E. Sanders, Phys. Rev. B 39, 1586 (1989); V. Privman, Phys. Rev. Lett. 69, 3686 (1992).

[7] C. Uebing and R. Gomer, J. Chem. Phys. 95, 7648 (1991).

[8] R. B. Pandey, Physica (Amsterdam) 187A, 77 (1992).

[9] B. Schmittmann, K. Hwang, and R. K. P. Zia, Europhys. Lett. 19, 19 (1992); K.-t. Leung, B. Schmittmann, and R. K. P. Zia, Phys. Rev. Lett. 62, 1772 (1989); B. Schmittmann and R. K. P. Zia, ibid. 66, 357 (1991).

[10] S. Katz, J. L. Lebowitz, and H. Spohn, Phys. Rev. B 28, 1655 (1983).

[11] J. Krug, Phys. Rev. Lett. 67, 1882 (1991).

[12] H. J. Bussemaker and M. H. Ernst, J. Stat. Phys. 68, 431 (1992).

[13] R. B. Pandey, Phys. Rev. A 42, 3363 (1990); R. B. Pandey and S. Gao, Phys. Rev. A 43, 4365 (1991).

[14] N. Metropolis, A. W. Rosenbluth, M. M. Rosenbluth, A. H. Teller, and E. Teller, J. Chem. Phys. 21, 1087 (1953).

[15] D. P. Landau, Phys. Rev. B 16, 117 (1977); 27, 5604 (1983).

[16] K. Kawasaki, in Phase Transitions and Critical Phenomena, edited by C. Domb and M. S. Green (Academic, New York, 1972).

[17] H. van Beijeren, J. Stat. Phys. 63, 47 (1991).

[18] D. Stauffer and A. Aharony, Introduction to Percolation Theory (Taylor and Francis, London 1992). 